\title{
Efficient Computation of Nonparaxial Surface Fields Excited on an Electrically Large Circular Cylinder With an Impedance Boundary Condition
}

\author{
Burak Alisan, Vakur B. Ertürk, Member, IEEE, and Ayhan Altintas, Senior Member, IEEE
}

\begin{abstract}
An alternative numerical approach is presented for the evaluation of the Fock-type integrals that exist in the uniform geometrical theory of diffraction (UTD)-based asymptotic solution for the nonparaxial surface fields excited by a magnetic or an electric source located on the surface of an electrically large circular cylinder with an impedance boundary condition (IBC). This alternative approach is based on performing numerical integration of the Fock-type integrals on a deformed path on which the integrands are nonoscillatory and rapidly decaying. Comparison of this approach with the previously developed one presented in [1], which is based on invoking the Cauchy's residue theorem by finding the pole singularities numerically, reveals that the alternative approach is considerably more efficient.
\end{abstract}

Index Terms-Fock-type integrals, impedance cylinder, surface fields, UTD-based Green's functions.

\section{INTRODUCTION}

$\mathbf{M}$ ANY military and commercial applications (e.g., missiles, mobile base stations, transreceivers of multipleinput multiple-output systems that might be mounted on curved host platforms, etc.) have stringent aerodynamic constraints that require the use of antennas that conform to their host platforms. This necessitates the development of efficient and accurate design and analysis tools for this class of antennas. Therefore, surface fields, created by a current distribution on the surface of a thin material coated (lossy or lossless) perfect electric conducting (PEC) circular cylinder, have been studied extensively using an impedance boundary condition (IBC). Analysis of slot/ aperture antennas as well as antennas on partially coated host platforms are typical applications that require the fast and accurate evaluation of these surface fields. Furthermore, the study of these surface fields may act as a canonical problem useful toward the development of asymptotic solutions valid for arbitrary smooth convex thin material coated/partially material coated surfaces [2], [3].

High-frequency-based asymptotic solutions for the surface fields on a source excited PEC convex surface have been investigated previously [4]-[10]. However, the study of surface fields

Manuscript received October 10, 2005; revised April 7, 2006. This work was supported by the Turkish Scientific and Technical Research Agency (TÜBITAK) under Grants EEEAG-104E044 and EEEAG-105E065.

B. Alisan is with the Department of Electrical and Electronics Engineering, Bilkent University, TR-06800 Bilkent, Ankara, Turkey. He is also with the Aselsan Electronics Inc., Ankara, Turkey.

V. B. Ertürk and A. Altintas are with the Department of Electrical and Electronics Engineering, Bilkent University, TR-06800 Bilkent, Ankara, Turkey.

Digital Object Identifier 10.1109/TAP.2006.880742 created by a current distribution on the surface of an impedance circular cylinder, which can also model a thin (lossy/lossless) material coated PEC case [11], is still a challenging problem. Recently, several high-frequency-based asymptotic solutions for the surface fields on a source excited circular cylinder with an IBC have been presented valid away from the paraxial region and within the paraxial region [1], [12]-[15]. Among them, the uniform geometrical theory of diffraction (UTD)-based asymptotic solution for a three-dimensional geometry [1], [13], [14] (valid away from the paraxial region) involves some Fock-type integrals and their derivatives which have to be evaluated numerically. However, special care is required in the computation of these integrals since the efficiency and accuracy of the overall solution strongly depend on the numerical evaluation of these integrals. In [1] (and in [13] and [14]), these Fock-type integrals have been evaluated by invoking the Cauchy's residue theorem, which requires finding the corresponding pole singularities numerically. It is claimed that the residue contributions coming from the first 20 poles yield sufficient accuracy.

Keeping this issue in mind, in this paper, an alternative numerical approach is offered for the evaluation of the Fock-type integrals (and their derivatives) that is based on performing a numerical integration along a deformed path. On this path, the Fock-type integrals exhibit a nonoscillatory and rapidly decaying nature. Hence, using a simple Gaussian quadrature algorithm is enough to obtain very accurate results efficiently. Consequently, this alternative approach is easier to implement and requires less computational time compared to the approach presented in [1]. It should be noted that the concept of performing a numerical integration on similar deformed integration paths has been previously used for the evaluation of surface fields of source excited electrically large dielectric coated circular cylinders in [16]-[19], and accurate results have been obtained.

Finally, for the sake of completeness, the UTD-based surface fields due to a tangential electric current source, which are valid away from the paraxial region, are also derived using an IBC and evaluated both performing an integration along the aforementioned deformed path and invoking the Cauchy's residue theorem (similar to [1]), whereas in [1] only the magnetic source case was considered.

The organization of this paper is as follows. In Section II, the UTD-based asymptotic solutions for the surface fields excited by both a magnetic and an electric source located on the surface of an electrically large impedance cylinder are given. The numerical evaluation of these surface fields is discussed in 


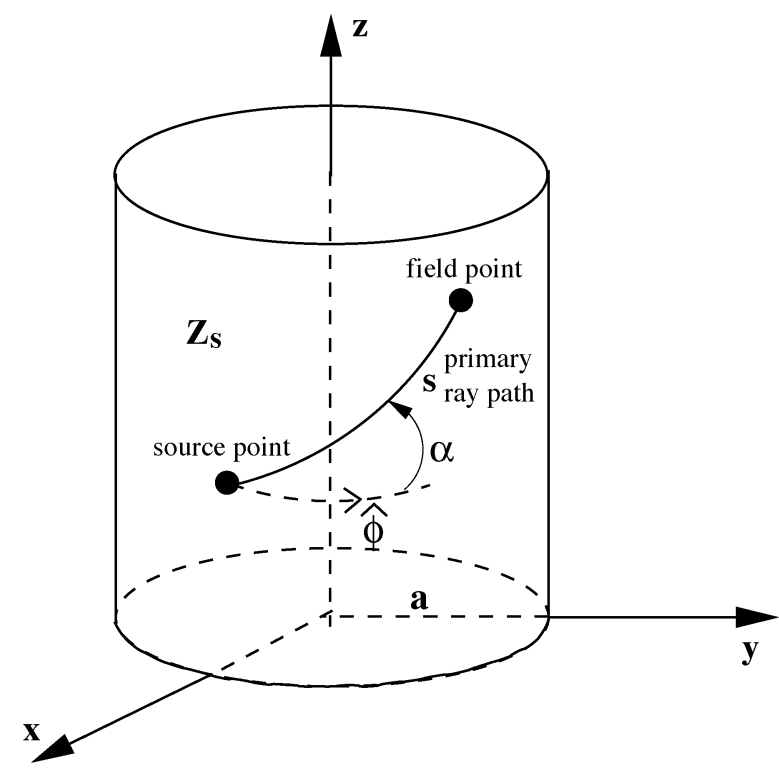

Fig. 1. Geometry of a circular cylinder with a radius $a$.

Section III, which presents a review of the approach presented in [1], and a detailed description of the alternative approach, namely, the numerical integration along a deformed integration path. In Section IV, several numerical results for the surface fields due to a tangential source (both magnetic and electric) are obtained using the two aforementioned approaches and compared with an eigenfunction solution to assess their accuracy and efficiency. An $e^{j w t}$ time dependence is assumed and suppressed throughout this paper.

\section{UTD SOLUTION}

Consider an electrically large circular cylinder with an IBC as shown in Fig. 1. The cylinder has a radius $a$ and a uniform surface impedance $Z_{s}$, and is assumed to be infinitely long along its axial direction.

\section{A. Magnetic Source}

For such a cylinder, the tangential surface field excited by a tangential magnetic source

$$
\vec{P}_{m}=P_{m}^{z} \hat{z}+P_{m}^{\phi} \hat{\phi}
$$

located on the surface is expressed in [1] as

$$
\vec{H}_{t}=\vec{P}_{m} \cdot\left(\hat{z}^{\prime} \hat{z} G_{z z}^{m}+\hat{\phi}^{\prime} \hat{z} G_{z \phi}^{m}+\hat{z}^{\prime} \hat{\phi} G_{\phi z}^{m}+\hat{\phi}^{\prime} \hat{\phi} G_{\phi \phi}^{m}\right)
$$

where $\vec{P}_{m}$ represents the strength and the orientation of the magnetic current and $G_{p q}^{m}$ is a UTD-based Green's function representation for a $\hat{p}(\hat{p}=\hat{z}$ or $\hat{\phi})$ oriented surface magnetic field due to a $\hat{q}(\hat{q}=\hat{z}$ or $\hat{\phi})$ directed magnetic current. Note that by relating the magnetic current to the magnetic field as in (2), the Green's function representation $G_{p q}^{m}$ is defined to have a unit of
$1 /\left(m^{2} \Omega\right)$. In (2), $G_{p q}^{m}$ represents the summation of all ray encirclements around the cylinder and can be determined as

$$
G_{p q}^{m}=\sum_{\ell=0}^{\infty}\left(G_{p q}^{m_{\ell+}}+G_{p q}^{m_{\ell-}}\right)
$$

where $G_{p q}^{m_{\ell+}}$ pertains to the Green's function which is responsible from the surface waves propagating around the cylinder in the positive $\hat{\phi}$ direction, whereas $G_{p q}^{m_{\ell-}}$ corresponds to those propagating in the negative $\hat{\phi}$ direction. Consequently, the UTDbased asymptotic Green's function representations for various source and field orientations are given in [1]

$$
\begin{aligned}
& G_{z z}^{m_{\ell \pm}} \sim G_{0}\left\{\cos ^{2} \alpha V_{0}+\frac{j}{k s}\left(1-\frac{j}{k s}\right)\left(2-3 \cos ^{2} \alpha\right) V_{0}\right. \\
& +\left[\frac{j}{3 k s}\left(1-\frac{j}{k s}\right) \sin ^{2} \alpha-\frac{\cos 2 \alpha}{36 k^{2} s^{2}}\right] V_{1} \\
& \left.+\frac{\sin ^{2} \alpha}{36 k^{2} s^{2}} V_{2}\right\} \\
& G_{z \phi}^{m_{\ell \pm}} \sim \mp G_{0}\left\{\cos \alpha \sin \alpha\left[1-\frac{j 3}{k s}\left(1-\frac{j}{k s}\right)\right] Y_{0}\right. \\
& +\left[\frac{j}{3 k s}\left(\tan ^{2} \alpha+\frac{j}{k s}\right) \cos \alpha \sin \alpha\right. \\
& \left.-\frac{\tan \alpha \cos 2 \alpha}{6 k^{2} s^{2}}\right] Y_{1} \\
& \left.+\frac{\tan \alpha \sin ^{2} \alpha}{36 k^{2} s^{2}} Y_{2}\right\} \\
& G_{\phi z}^{m_{\ell \pm}} \sim \mp G_{0}\left\{\cos \alpha \sin \alpha\left[X_{0}+V_{0}-\frac{j 3}{k s}\left(1-\frac{j}{k s}\right) V_{0}\right]\right. \\
& +\left[\frac{j}{k}\left(1-\frac{j}{k s}\right)\left(\frac{2 \tan \alpha}{3 s}-\frac{\cos \alpha \sin \alpha}{3 s}\right)\right. \\
& \left.-\frac{\sin 2 \alpha}{6 k^{2} s^{2}}\right] V_{1}-\frac{\sin \alpha}{36 s^{2}} \\
& \left.\times\left(\cos \alpha-\frac{4}{\cos \alpha}\right) V_{2}\right\} \\
& G_{\phi \phi}^{m_{\ell \pm}} \sim G_{0}\left\{\sin ^{2} \alpha Y_{0}+\frac{j}{k s}\left(1-\frac{j}{k s}\right)\left(2-3 \sin ^{2} \alpha\right) Y_{0}\right. \\
& +\frac{j}{k s} \frac{1}{\cos ^{2} \alpha}\left(U_{0}-Y_{0}\right) \\
& +\left[\frac{j}{k s}\left(1-\frac{j}{k s}\right)\left(\frac{\cos ^{2} \alpha-\sin ^{2} \alpha-4}{6 s}\right)\right. \\
& -\frac{j}{6 s}\left(\cos \alpha-\frac{4}{\cos \alpha}-\frac{j}{k s} \tan \alpha \sin \alpha\right) \\
& \left.-\frac{\tan \alpha \sin 2 \alpha}{6 k^{2} s^{2}}\right] Y_{1} \\
& \left.-\left(\frac{\sin ^{2} \alpha+4 \tan ^{2} \alpha}{36 k^{2} s^{2}}\right) Y_{2}\right\}
\end{aligned}
$$

where $G_{0}=-\left(j k e^{-j k s} / 2 \pi Z_{0} s\right), k$ is the free space wave number, $Z_{0}$ is the free space impedance, $s$ is the distance along the geodesic ray path, and $\alpha$ is the angle between $s$ and the positive $\hat{\phi}$ direction as shown in Fig. 1. It should be mentioned that 
the expressions given in (4)-(7) are valid in the nonparaxial region and developed mainly for large separations $s$ between the source and field points. However, since some of the secondorder terms in $s$ are included, they may remain accurate even for relatively small separations.

The $U_{0}, X_{0}, V_{0}, Y_{0}, V_{1}, Y_{1}, V_{2}$, and $Y_{2}$ terms in (4)-(7) are expressed in [1] in terms of simpler Fock-type integrals in the form of

$$
\Upsilon_{r}=\int_{-\infty}^{\infty} d \tau e^{-j \xi \tau} \frac{\left(R_{w}\right)^{r}}{D_{w}} \quad r=0,1,2
$$

where

$$
\begin{aligned}
D_{w} & =\left(R_{w}-q_{e}\right)\left(R_{w}-q_{m}\right)+q_{c}^{2} \\
q_{e} & =-j m_{t} \Lambda \cos \alpha \\
q_{m} & =-j m_{t} \Lambda^{-1} \cos \alpha \\
q_{c} & =-j m_{t}\left(1+\frac{\tau}{2 m_{t}^{2}}\right) \sin \alpha \\
R_{w} & =W_{2}^{\prime}(\tau) / W_{2}(\tau)
\end{aligned}
$$

in which $W_{2}(\tau)$ is a Fock-type Airy function and $W_{2}^{\prime}(\tau)$ is its derivative with respect to $\tau$. In addition, $\Lambda=Z_{s} / Z_{0}$ is the normalized surface impedance, $m_{t}=\left(k_{\rho} a / 2\right)^{1 / 3}, \xi=m_{t} \phi_{\ell}^{ \pm}$, $\phi_{\ell}^{ \pm}= \pm\left(\phi-\phi^{\prime}-\pi\right)+(2 \ell+1) \pi, k_{z}$, and $k_{\rho}$ are the axial and radial wave numbers, respectively, such that

$$
k_{\rho}=\left\{\begin{array}{ll}
\sqrt{k^{2}-k_{z}^{2}}, & \text { if } k^{2} \geq k_{z}^{2} \\
-j \sqrt{k_{z}^{2}-k^{2}}, & \text { if } k^{2}<k_{z}^{2}
\end{array} .\right.
$$

The simplified equations are, in turn, given in [1] as follows:

$$
\begin{aligned}
U_{0} & =-j \xi q_{m} \sqrt{\frac{j \xi}{\pi}}\left(\Upsilon_{2}-q_{e} \Upsilon_{1}\right) \\
X_{0} & =-\frac{1}{2} \sqrt{\frac{j \xi}{\pi}}\left(\Upsilon_{1}+\frac{j}{2 m_{t}^{2}} \frac{\partial \Upsilon_{1}}{\partial \xi}\right) \\
V_{0} & =\frac{1}{2} \sqrt{\frac{j \xi}{\pi}}\left(\Upsilon_{1}-q_{m} \Upsilon_{0}\right) \\
Y_{0} & =-\frac{q_{m}}{2} \sqrt{\frac{j \xi}{\pi}}\left(\Upsilon_{0}+\frac{j}{2 m_{t}^{2}} \frac{\partial \Upsilon_{0}}{\partial \xi}\right) \\
V_{1} & =\frac{1}{2} \sqrt{\frac{j \xi}{\pi}}\left[\Upsilon_{1}-q_{m} \Upsilon_{0}+2 \xi\left(\frac{\partial \Upsilon_{1}}{\partial \xi}-q_{m} \frac{\partial \Upsilon_{0}}{\partial \xi}\right)\right] \\
Y_{1} & =-\frac{q_{m}}{2} \sqrt{\frac{j \xi}{\pi}}\left[\Upsilon_{0}+\left(\frac{j}{2 m_{t}^{2}}+2 \xi\right) \frac{\partial \Upsilon_{0}}{\partial \xi}\right] \\
V_{2} & =\frac{1}{2} \sqrt{\frac{j \xi}{\pi}}\left[3\left(\Upsilon_{1}-q_{m} \Upsilon_{0}\right)+8 \xi\left(\frac{\partial \Upsilon_{1}}{\partial \xi}-q_{m} \frac{\partial \Upsilon_{0}}{\partial \xi}\right)\right] \\
Y_{2} & =-\frac{q_{m}}{2} \sqrt{\frac{j \xi}{\pi}}\left[3 \Upsilon_{0}+\left(\frac{j 3}{2 m_{t}^{2}}+8 \xi\right) \frac{\partial \Upsilon_{0}}{\partial \xi}\right] .
\end{aligned}
$$

\section{B. Electric Source}

For the sake of completeness, using a formulation similar to the procedure presented in [1], the tangential surface field ex- cited by a tangential electric source can be derived for the same geometry. First, $\hat{z}$ components of electric and magnetic fields due to a tangential electric source

$$
\vec{P}_{e}=P_{e}^{z} \hat{z}+P_{e}^{\phi} \hat{\phi}
$$

located on the surface are derived as

$$
\begin{aligned}
E_{z}= & -\frac{Z_{s}}{4 \pi^{2} a} \int_{-\infty}^{\infty} d k_{z} e^{-j k_{z} z_{d}} \\
& \times \sum_{n=-\infty}^{\infty} \frac{e^{j n \phi_{d}}}{D_{c}}\left[\left(1+\frac{j \Lambda^{-1} k}{k_{\rho}} R_{n}\right) P_{e}^{z}+\frac{n k_{z}}{k_{\rho}^{2} a} P_{e}^{\phi}\right] \\
& \times \frac{H_{n}^{(2)}\left(k_{\rho} \rho\right)}{H_{n}^{(2)}\left(k_{\rho} a\right)} \\
H_{z}= & \frac{1}{4 \pi^{2} a} \int_{-\infty}^{\infty} d k_{z} e^{-j k_{z} z_{d}} \\
& \times \sum_{n=-\infty}^{\infty} \frac{e^{j n \phi_{d}}}{D_{c}}\left[\frac{n k_{z}}{k_{\rho}^{2} a} P_{e}^{z}+\left(1+\frac{j \Lambda k}{k_{\rho}} R_{n}\right) P_{e}^{\phi}\right] \\
& \times \frac{H_{n}^{(2)}\left(k_{\rho} \rho\right)}{H_{n}^{(2)}\left(k_{\rho} a\right)}
\end{aligned}
$$

where

$$
\begin{aligned}
R_{n} & =\frac{H_{n}^{(2)^{\prime}}\left(k_{\rho} a\right)}{H_{n}^{(2)}\left(k_{\rho} a\right)} \\
D_{c} & =\left(1+\frac{j \Lambda k}{k_{\rho}} R_{n}\right)\left(1+\frac{j \Lambda^{-1} k}{k_{\rho}} R_{n}\right)-\left(\frac{n k_{z}}{k_{\rho}^{2} a}\right)^{2}
\end{aligned}
$$

and

$$
z_{d}=z-z^{\prime} \quad \phi_{d}=\phi-\phi^{\prime} .
$$

As expected, the $\hat{z}$ components of the fields $\left(E_{z}, H_{z}\right)$ are the dual of the $\hat{z}$ components of the fields obtained for the magnetic source case in [1].

Once the $\hat{z}$ components of the fields $\left(E_{z}, H_{z}\right)$ are obtained, the vector potentials $\left(A_{z}, F_{z}\right)$ due to these components can easily be found using the methods described in [20]. Then, the procedure explained in [1] is followed. Namely, first Watson transform is applied to the potentials and thereby the potentials are expressed as double integrals over axial $\left(k_{z}\right)$ and azimuthal $(\nu)$ wavenumbers. Employing a Fock substitution $\left(\nu=k_{\rho} a+m_{t} \tau\right)$, integration in the $\nu$-plane is replaced by integration in the $\tau$-plane. Then, introducing a standard polar transformation along with some geometrical relations, integration over $k_{z}$ is converted to a complex contour integral, which is evaluated applying the method of steepest descent assuming that the separation $s$ between the source and field points is a large parameter. Finally, field expressions are obtained by performing the derivatives to the resultant potential expressions analytically (where the terms including higher powers of $m_{t}^{-2}$ 
are neglected). As a result, the tangential surface field excited by a tangential electric source given by (23) is expressed as

$$
\vec{H}_{t}=\vec{P}_{e} \cdot\left(\hat{z}^{\prime} \hat{z} G_{z z}^{e}+\hat{\phi}^{\prime} \hat{z} G_{z \phi}^{e}+\hat{z}^{\prime} \hat{\phi} G_{\phi z}^{e}+\hat{\phi}^{\prime} \hat{\phi} G_{\phi \phi}^{e}\right)
$$

where $G_{p q}^{e}$ is a UTD-based Green's function representation for a $\hat{p}(\hat{p}=\hat{z}$ or $\hat{\phi})$ oriented surface magnetic field due to a $\hat{q}(\hat{q}=\hat{z}$ or $\hat{\phi}$ ) directed electric current. Note that by relating the electric current to the magnetic field as in (29), the Green's function representation $G_{p q}^{e}$ is defined to have a unit of $1 / \mathrm{m}^{2}$. Similar to the magnetic case in (29), $G_{p q}^{e}$ contains the summation of all ray encirclements around the cylinder. Finally, the explicit expressions for the UTD-based asymptotic Green's function representations for various source and field orientations for the electric case are given by

$$
\begin{aligned}
& G_{z z}^{e_{\ell \pm}} \sim \pm Z_{s} G_{0}\left\{\cos \alpha \sin \alpha\left[1-\frac{j 3}{k s}\left(1-\frac{j}{k s}\right)\right] Y_{0}\right. \\
& +\left[\frac{j}{3 k s}\left(\tan ^{2} \alpha+\frac{j}{k s}\right) \cos \alpha \sin \alpha\right. \\
& \left.-\frac{\tan \alpha \cos 2 \alpha}{6 k^{2} s^{2}}\right] Y_{1} \\
& \left.+\frac{\tan \alpha \sin ^{2} \alpha}{36 k^{2} s^{2}} Y_{2}\right\} \\
& G_{z \phi}^{e_{\ell \pm}} \sim Z_{s} G_{0}\left\{\cos ^{2} \alpha V_{0}+\frac{j}{k s}\left(1-\frac{j}{k s}\right)\left(2-3 \cos ^{2} \alpha\right) V_{0}\right. \\
& +\left[\frac{j}{3 k s}\left(1-\frac{j}{k s}\right) \sin ^{2} \alpha-\frac{\cos 2 \alpha}{36 k^{2} s^{2}}\right] V_{1} \\
& \left.+\frac{\sin ^{2} \alpha}{36 k^{2} s^{2}} V_{2}\right\} \\
& G_{\phi z}^{e_{\ell \pm}} \sim-Z_{s} G_{0}\left\{\sin ^{2} \alpha Y_{0}+\frac{j}{k s}\left(1-\frac{j}{k s}\right)\left(2-3 \sin ^{2} \alpha\right) Y_{0}\right. \\
& +\frac{j}{k s} \frac{1}{\cos ^{2} \alpha}\left(U_{0}-Y_{0}\right) \\
& +\left[\frac{j}{k s}\left(1-\frac{j}{k s}\right)\left(\frac{\cos ^{2} \alpha-\sin ^{2} \alpha-4}{6 s}\right)\right. \\
& -\frac{j}{6 s}\left(\cos \alpha-\frac{4}{\cos \alpha}-\frac{j}{k s} \tan \alpha \sin \alpha\right) \\
& \left.-\frac{\tan \alpha \sin 2 \alpha}{6 k^{2} s^{2}}\right] Y_{1} \\
& \left.-\left(\frac{\sin ^{2} \alpha+4 \tan ^{2} \alpha}{36 k^{2} s^{2}}\right) Y_{2}\right\} \\
& G_{\phi \phi}^{e_{\ell \pm}} \sim \mp Z_{s} G_{0}\left\{\cos \alpha \sin \alpha\left[X_{0}+V_{0}-\frac{j 3}{k s}\left(1-\frac{j}{k s}\right) V_{0}\right]\right. \\
& +\left[\frac{j}{k}\left(1-\frac{j}{k s}\right)\left(\frac{2 \tan \alpha}{3 s}-\frac{\cos \alpha \sin \alpha}{3 s}\right)\right. \\
& \left.-\frac{\sin 2 \alpha}{6 k^{2} s^{2}}\right] V_{1} \\
& \left.-\frac{\sin \alpha}{36 s^{2}}\left(\cos \alpha-\frac{4}{\cos \alpha}\right) V_{2}\right\}
\end{aligned}
$$

where $U_{0}, X_{0}, V_{0}, Y_{0}, V_{1}, Y_{1}, V_{2}$, and $Y_{2}$ are the same functions given by (15)-(22). It should be mentioned that if $G_{p q}^{e}$ was defined to relate the electric current density to the electric field (i.e., the left-hand side of (29) would be $\vec{E}_{t}$ ), then $G_{p q}^{e}$ could also be determined via duality.

Similar to the magnetic source case, expressions given in (30)-(33) are valid in the nonparaxial region and developed mainly for large separations between the source and field points. However, they may also remain accurate for relatively small separations due to the second-order terms in $s$.

\section{NumERICAL EVALUATION OF SURFACE FIELDS}

The major difficulty in the evaluation of (4)-(7) and (30)-(33) is the numerical evaluation of the Fock-type integrals given in (8). Since the accuracy and efficiency of the surface fields strongly depend on these integrals, special care is required for their numerical evaluation. Therefore, in this section, the approach presented in [1] is briefly reviewed (as residue series approach), and then the alternative approach (numerical integration approach) is presented.

\section{A. Residue Series Approach [1]}

This approach is based on invoking the Cauchy's residue theorem for the evaluation of the Fock-type integrals. The values of integrals are obtained by summing the residues at the pole singularities of the integrands. The poles, which are the roots of the denominator of the integrands given in (8), should be determined first. To determine these roots, $D_{w}$ is written in the following form:

$$
D_{w}=Q_{e}(\tau) Q_{m}(\tau)
$$

where

$$
\begin{aligned}
Q_{e}(\tau) & =R_{w}-q_{e}+\sigma \\
Q_{m}(\tau) & =R_{w}-q_{m}-\sigma \\
\sigma & =\frac{q_{e}-q_{m}-\sqrt{\left(q_{e}-q_{m}\right)^{2}-4 q_{c}^{2}}}{2}
\end{aligned}
$$

with the root having the positive real part is chosen. The roots of $Q_{e}$, namely, $\tau_{p i_{1}}$, are determined by applying the Newton-Raphson method. In this method, an initial estimate for the locations of the roots is required, and this estimate must be close to the original location of the roots. For this reason, as an initial estimate, the roots of $\left(R_{w}-q_{e}\right)$ are obtained. Then, the roots are tracked from $\left(R_{w}-q_{e}\right)$ to $Q_{e}$ using a step by step procedure as described in [1]. The roots of $Q_{m}$, namely, $\tau_{p i_{2}}$, are determined in a similar manner. Finally, using Cauchy's residue theorem, the Fock-type integral in (8) is represented as follows:

$$
\begin{aligned}
\Upsilon_{r}=-j 2 \pi \sum_{i=1}^{\infty}\left[e^{-j \xi \tau}\right. & \left.\frac{\left(R_{w}\right)^{r}}{Q_{e}^{\prime}(\tau) Q_{m}(\tau)}\right|_{\tau=\tau_{p i_{1}}} \\
& \left.+\left.e^{-j \xi \tau} \frac{\left(R_{w}\right)^{r}}{Q_{e}(\tau) Q_{m}^{\prime}(\tau)}\right|_{\tau=\tau_{p i_{2}}}\right]
\end{aligned}
$$




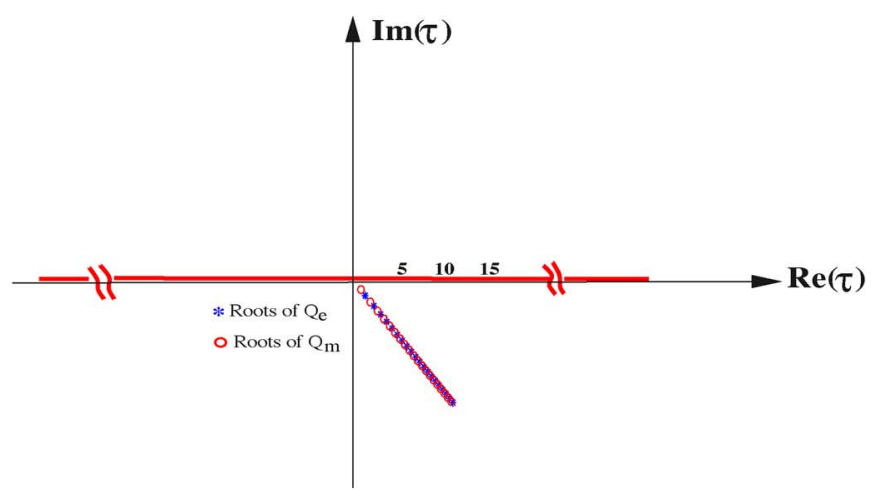

(a)

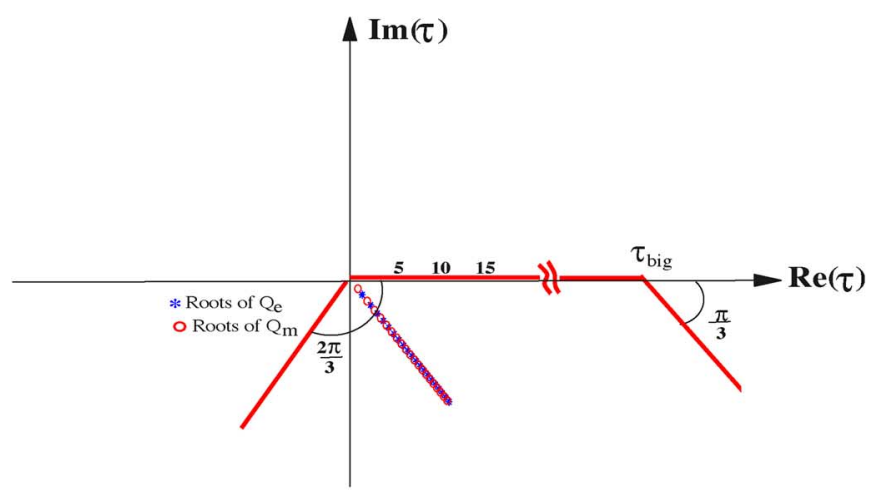

(b)

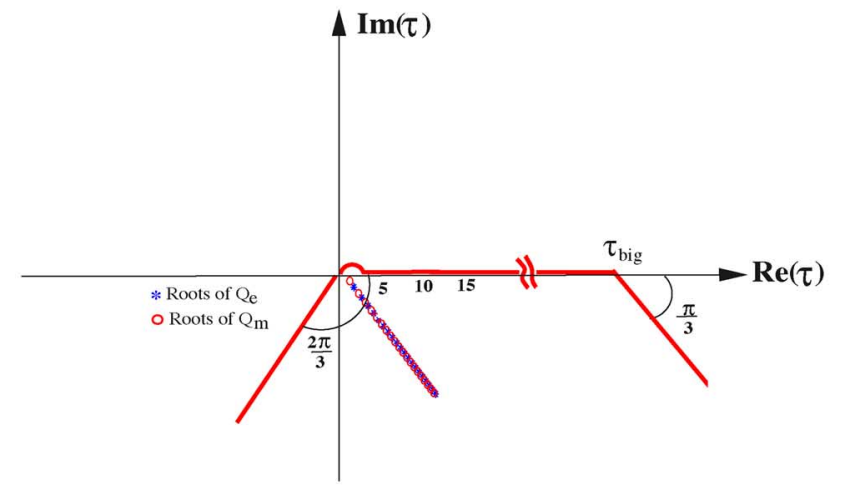

(c)

Fig. 2. Paths of integration (a) Original path. (b) Deformed path 1. (c) Deformed path 2, used when the dominant pole is very close to the integration path (like an Elliott mode). (Color version available online at http://ieeexplore. ieee.org.)

The first 20 roots (20 for $Q_{e}$ and 20 for $Q_{m}$ ) are included to obtain accurate results as suggested in [1].

\section{B. Numerical Integration Approach}

This approach is based on performing a numerical integration for the evaluation of the Fock-type integrals. The original integration path for the Fock-type integrals given by (8) ranges from $-\infty$ to $\infty$ on the complex $\tau$-plane, as shown in Fig. 2(a), where $\tau$ is the integration variable. Unfortunately, the integrals may not converge rapidly when this path is used since the integrands have a highly oscillatory and slowly convergent behavior. This is illustrated in Fig. 3, where the variation of the real and imaginary parts of the integrand of a typical Fock-type
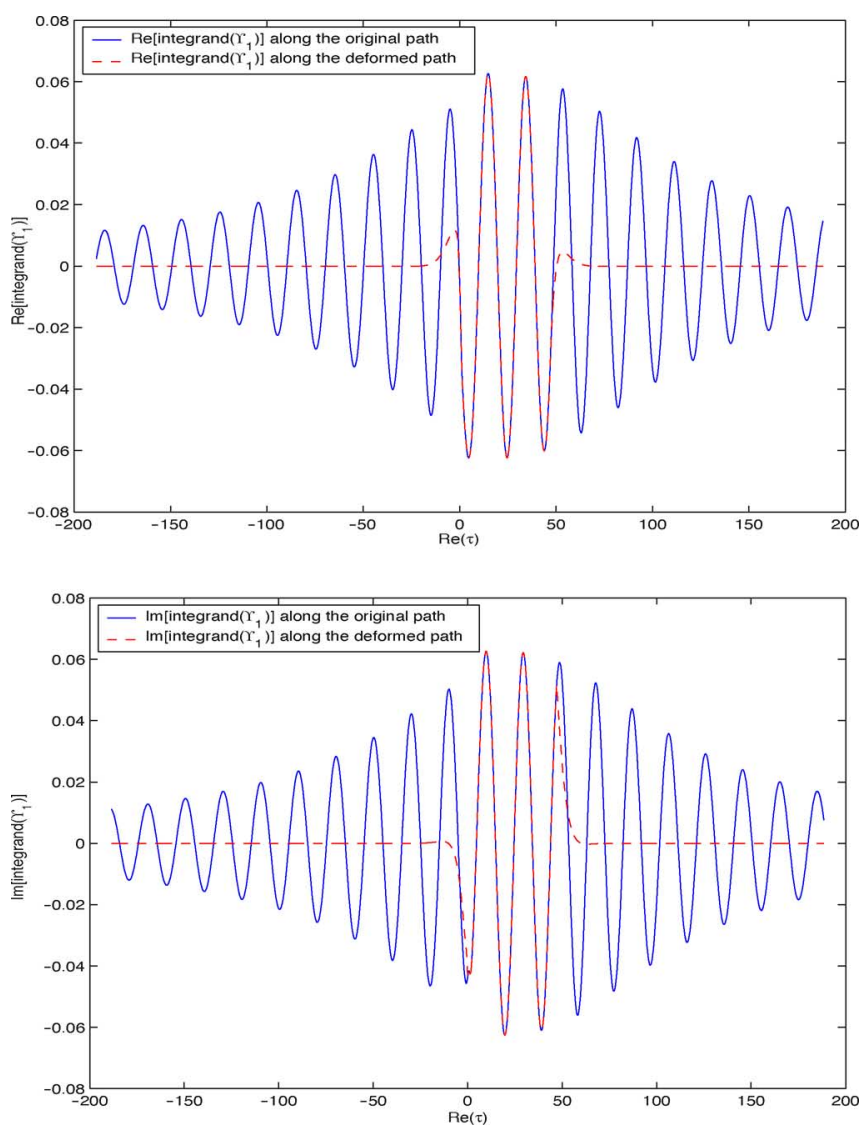

Fig. 3. (a) Real and (b) imaginary parts of integrand of a typical Fock-type integral (in this case integrand of $\Upsilon_{1}$ ) along the original and deformed paths. (Color version available online at http://ieeexplore.ieee.org.)

integral $\left(\Upsilon_{1}\right)$ versus $\tau$ is depicted. Therefore, these integrals are evaluated on a deformed path similar to the one in [18] and [19]. Note that various types of deformed paths have been previously used for the coated cylinder case in [16], [17], and [21]. However, the path suggested in [18] and [19] seems to yield the best result for the evaluation of the Fock-type integrals pertaining to a circular cylinder with an IBC. To obtain accurate results, the path deformation should be done carefully so that all pole singularities are captured. The poles are known to be in the second and fourth quadrants. The second quadrant poles are the negative of the fourth quadrant poles; only the fourth quadrant poles are shown in Fig. 2, where the poles of $\Upsilon_{1}$ pertaining to an electrically large cylinder with $a=5 \lambda, \Lambda=0.1$ at $7 \mathrm{GHz}$ are determined for $s=1 \lambda, \alpha=\pi / 4$. Since there is no pole in the third quadrant, part of the integration path ranging from $-\infty$ to zero can be safely deformed to the third quadrant. However, special attention is required in deforming the part of the integration path ranging from zero to $\infty$ into the fourth quadrant because of the existence of the pole singularities. As the pole locations in this quadrant are similar to [18] and [19], the critical issues manifest themselves in the location of the first (dominant) pole and in the slope of the pole location trajectories. It is seen that the dominant pole has the closest location to the integration path and may come very close to the real $\tau$-axis, thereby giving rise to a low-attenuation Elliott mode for some surface impedance values $Z_{s}$ [22]-[24]. Moreover, it has 
a real part significantly smaller than $\tau_{\text {big }}$ [defined in Fig. 2(b)]. On the other hand, all remaining poles, which can be defined as $\tau_{p_{i}}=\operatorname{Re}\left(\tau_{p_{i}}\right)-j \operatorname{Im}\left(\tau_{p_{i}}\right),(i=2,3,4, \ldots)$, are lined up on the fourth quadrant satisfying the following condition: $\operatorname{Re}\left(\tau_{p_{i}}\right)<\operatorname{Re}\left(\tau_{p_{i}+1}\right)$ and $\operatorname{Im}\left(\tau_{p_{i}}\right)<\operatorname{Im}\left(\tau_{p_{i}+1}\right)$, as shown in Fig. 2, and the slope of the pole location trajectory is approximately $\pi / 3$ (defined from the positive real $\tau$ axis). Note that when $|\tau|$ is very large, the trajectory approaches to $\pi / 2$ (similar to PEC cylinders) [25].

In the light of above considerations, the Fock-type integrals, whose generic form is given in (8), are split into three integrals ranging from $(-\infty, 0),\left(0, \tau_{\text {big }}\right)$, and $\left(\tau_{\text {big }}, \infty\right)$, where $\tau_{\text {big }}$ is chosen approximately $1.5 \mathrm{ka}$. Such a choice guarantees that all pole singularities corresponding to different cylinder size (varying between $3 \lambda$ and $6 \lambda$ ) and different cylinder surface impedance values (varying between $|\Lambda|=0.1$ and $|\Lambda|=5$ ) studied in this paper and in [1] are captured. Furthermore, as the frequency is increased, there will be relatively little change in the position of the poles that reside near the $e^{-j \pi / 3}$ axis in the $\tau$-plane. However, based on the location of the dominant pole, small adjustments can be done about the value of $\tau_{\text {big }}$ (even setting $\tau_{\text {big }}=k a$ captures all the poles for all cases studied in [1]). As the next step, the integration path for the first and third integrals are deformed to $\left(\infty e^{-j 2 \pi / 3}, 0\right)$, and $\left(\tau_{\mathrm{big}}, \infty e^{-j \pi / 3}\right)$, respectively. Then, the integration variable $\tau$ is changed to $\tau e^{j 2 \pi / 3}$ for the first integral and to $\left(\tau-\tau_{\text {big }}\right) e^{j \pi / 3}$ for the third integral, causing the Airy function and its derivative to be nonoscillatory and decay most rapidly (an exponential decay is achieved) as $|\tau| \rightarrow \infty$ along the path where $\arg (\tau)=0$ [21]. Consequently, the first and the third integrals now range from zero to $\infty$, they are fast decaying and nonoscillatory. This is shown in Fig. 3, where the variation of the real and imaginary parts of the integrand of the Fock-type integral $\Upsilon_{1}$ versus $\operatorname{Re}(\tau)$ (mentioned above) is plotted along the original and deformed paths for the aforementioned impedance cylinder (i.e., $a=5 \lambda, \Lambda=0.1, f=7 \mathrm{GHz}, s=1 \lambda$, and $\alpha=\pi / 4$ ). The value of the integrand (both real and imaginary parts) for the first and the third integrals exponentially decays and goes to zero. Although the integrand of the second integral is oscillatory, the integration interval is quite short and hence, its evaluation does not create a severe problem. Still, most of the $\mathrm{CPU}$ time is consumed during the computation of this second integral. Finally, all integrals can be integrated efficiently using a simple Gaussian quadrature algorithm. It should be noted that, in the case of a pole very close to the integration path, a small semicircle as shown in Fig. 2(c) is introduced.

\section{NumERICAL RESUlTS AND DISCUSSIONS}

To illustrate and compare the efficiency and accuracy of the aforementioned approaches, several numerical examples for surface fields due to both magnetic and electric sources are obtained using two different numerical approaches and compared with the eigenfunction solution. The eigenfunction solution for the surface fields due to the magnetic source is given in [1], and the solution due to the electric source is the dual of the magnetic source case.
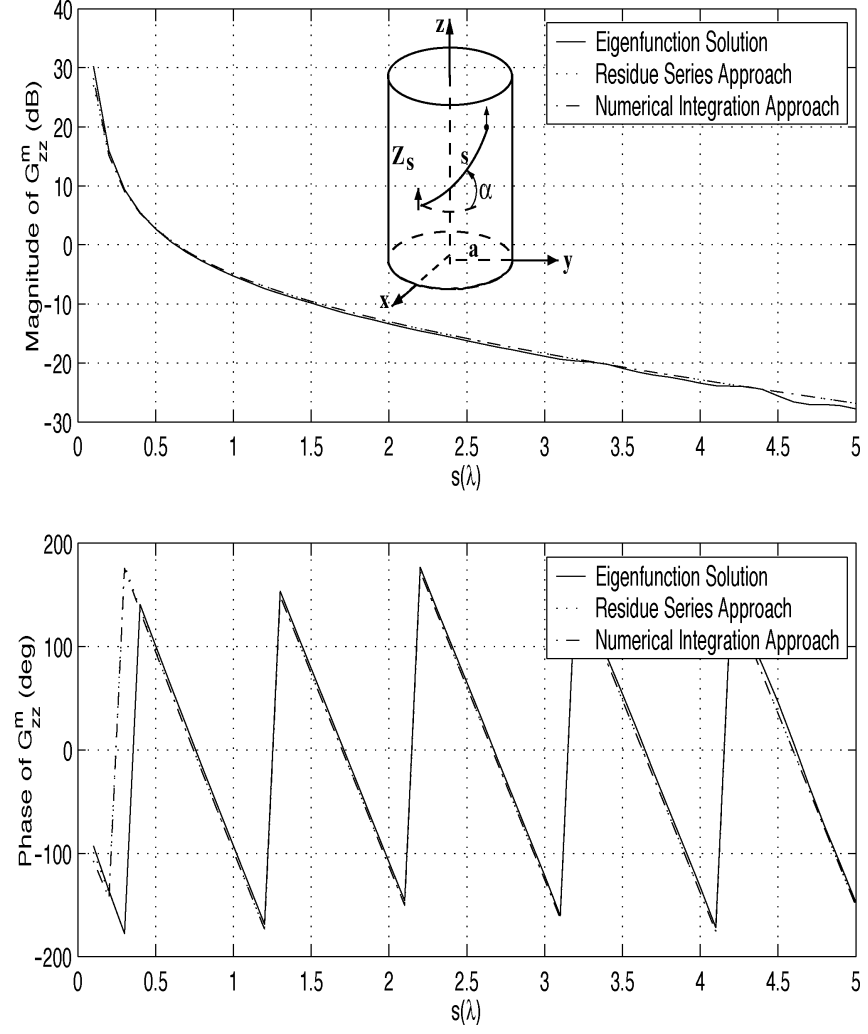

Fig. 4. Comparison of the magnitude (in $\mathrm{dB}$ ) and phase of the $G_{z z}^{m}$ versus separation $s$ obtained by the eigenfunction solution and the numerical approaches for $f=7 \mathrm{GHz}, a=5 \lambda, \alpha=45^{\circ}$, and $\Lambda=0.1$.

Various components of Green's function representations are computed for the geodesic path length varying from $0.1 \lambda$ to $5 \lambda$ at $f=7 \mathrm{GHz}$ on the aforementioned cylinder (with a radius $5 \lambda$ and a normalized surface impedance $\Lambda=0.1$ ) for a fixed azimuthal angle $\left(\alpha=45^{\circ}\right)$. The Fock-type integrals in the Green's function representations are evaluated by i) Cauchy's residue theorem (residue series approach) and ii) numerical integration approach by setting $\tau_{\text {big }}$ to $1.5 \mathrm{ka}$. Note that because the cylinder is electrically large $(a>1 \lambda)$, it is enough to retain the $\ell=0$ term [1], which corresponds to the primary rays propagating around the cylinder. Therefore, in all numerical examples presented, only the leading term is retained.

Components of the Green's function representation due to a magnetic source (i.e., $G_{p q}^{m}$ ) obtained by these approaches are plotted in Figs. 4-6. Both approaches yield a very good agreement when compared with the eigenfunction solution given in [1] as illustrated in Figs. 4-6. It should be noted, however, that the numerical integration has several advantages over the residue series approach. First, it is more efficient in terms of computational time, as shown in Table I. Secondly, locating the poles requires a difficult and a complex procedure. One can easily miss a pole, and/or pole search algorithms may need to be modified for some geometries and physical parameters. Finally, a finite number of poles are taken into consideration in residue series approach, whereas all poles are included during the numerical integration.

Also, for the same geometry considered above, various components of Green's function representations due to an electric 

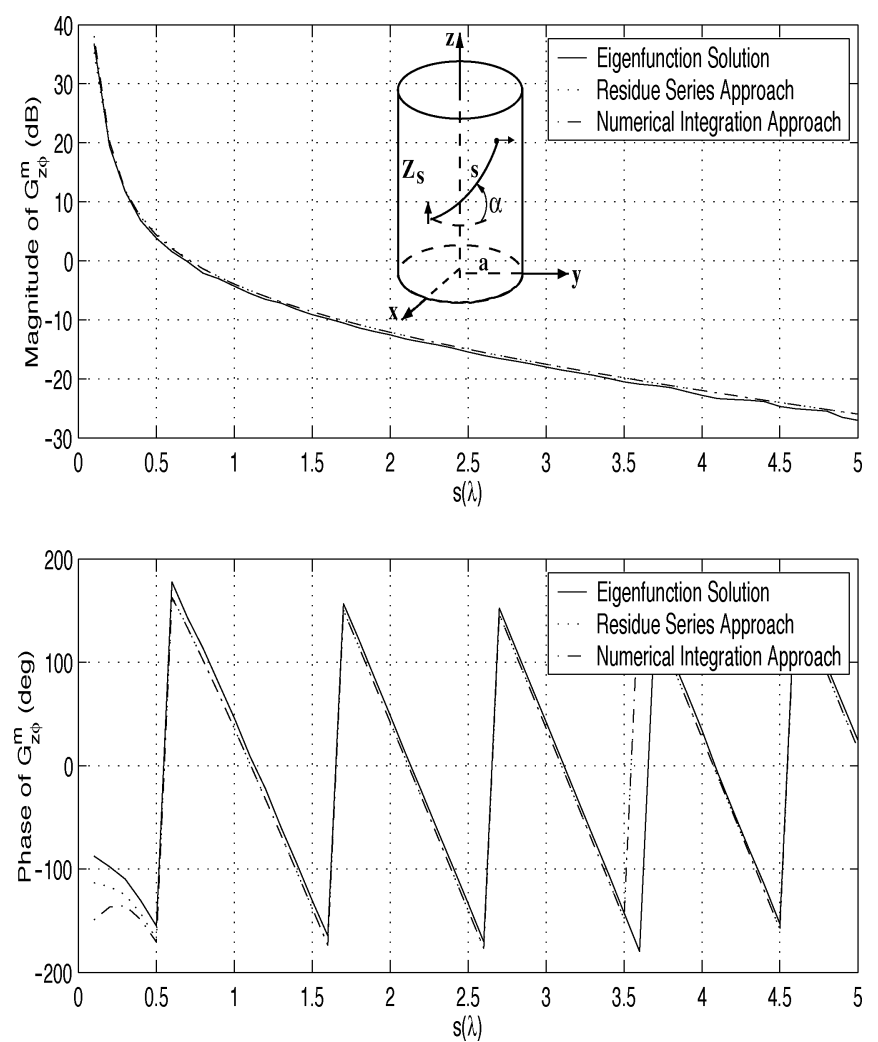

Fig. 5. Comparison of the magnitude (in dB) and phase of the $G_{z \phi}^{m}$ versus separation $s$ obtained by the eigenfunction solution and the numerical approaches for $f=7 \mathrm{GHz}, a=5 \lambda, \alpha=45^{\circ}$, and $\Lambda=0.1$.
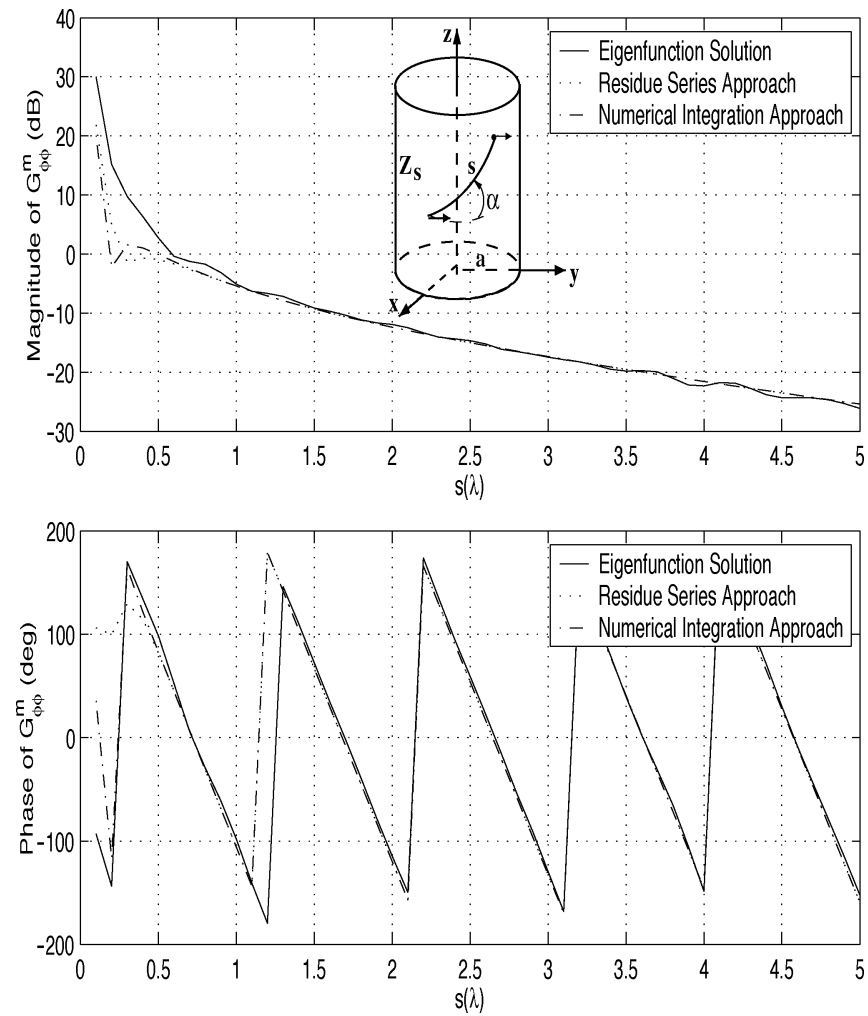

Fig. 6. Comparison of the magnitude (in dB) and phase of the $G_{\phi \phi}^{m}$ versus separation $s$ obtained by the eigenfunction solution and the numerical approaches for $f=7 \mathrm{GHz}, a=5 \lambda, \alpha=45^{\circ}$, and $\Lambda=0.1$.

source (i.e., $G_{p q}^{e}$ ) are computed using both approaches and compared with the eigenfunction solution in Figs. 7-9. Both ap-
TABLE I

COMPUTATIONAL TIME

\begin{tabular}{cccccc}
\hline \hline & \multicolumn{2}{c}{ Residue Series Approach } & & Numerical Integration Approach \\
\cline { 2 - 3 } \cline { 5 - 6 } & Mag. source & Elec. source & & Mag. source & Elec. source \\
$G_{z z}$ & $92.2 \mathrm{sec}$. & $89.5 \mathrm{sec}$. & & $19.8 \mathrm{sec}$. & $19.7 \mathrm{sec}$. \\
$G_{z \phi}$ & $89.1 \mathrm{sec}$. & $92.6 \mathrm{sec}$. & & $19.7 \mathrm{sec}$. & $19.8 \mathrm{sec}$. \\
$G_{\phi \phi}$ & $89.7 \mathrm{sec}$. & $89.6 \mathrm{sec}$. & & $29.6 \mathrm{sec}$. & $19.7 \mathrm{sec}$. \\
\hline
\end{tabular}
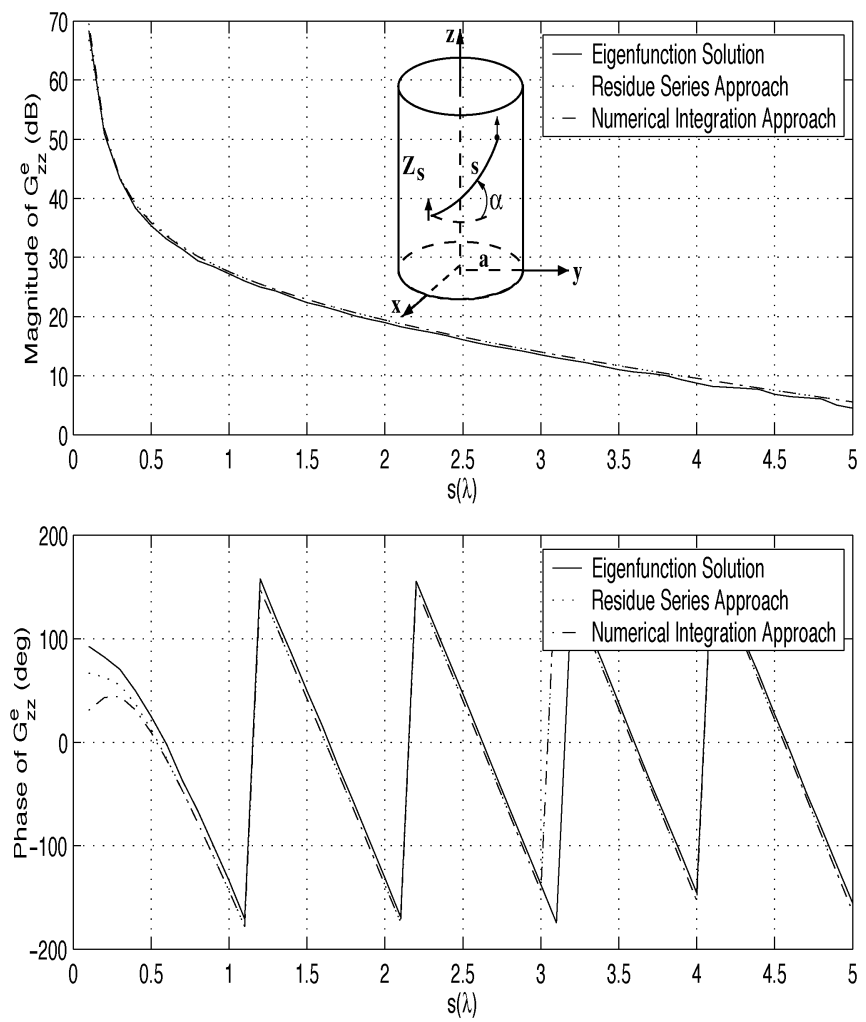

Fig. 7. Comparison of the magnitude (in $\mathrm{dB}$ ) and phase of the $G_{z z}^{e}$ versus separation $s$ obtained by the eigenfunction solution and the numerical approaches for $f=7 \mathrm{GHz}, a=5 \lambda, \alpha=45^{\circ}$, and $\Lambda=0.1$.

proaches are in very good agreement with the eigenfunction solution. However, similar to the magnetic source case, computation of surface fields using the numerical integration approach requires less CPU time as shown in Table I.

It should be noted that the developed UTD-based asymptotic Green's function representations (i.e., the surface fields) are derived for large separations between the source and field points. Therefore, the results are expected to be accurate for large separations. However, results obtained in this paper using the two approaches are accurate even for relatively small separations (though we do not expect them to be accurate near the source). Small disagreements between the eigenfunction solution and the two approaches are due to the convergence problem of the eigenfunction solution, which is expected especially for large separations and clearly seen in all numerical results. On the other hand, the eigenfunction solution for the $G_{\phi \phi}^{m}$ component is the most slowly convergent component, and such a slow convergence affects its agreement with the asymptotic solutions starting from approximately $0.5 \lambda$. 

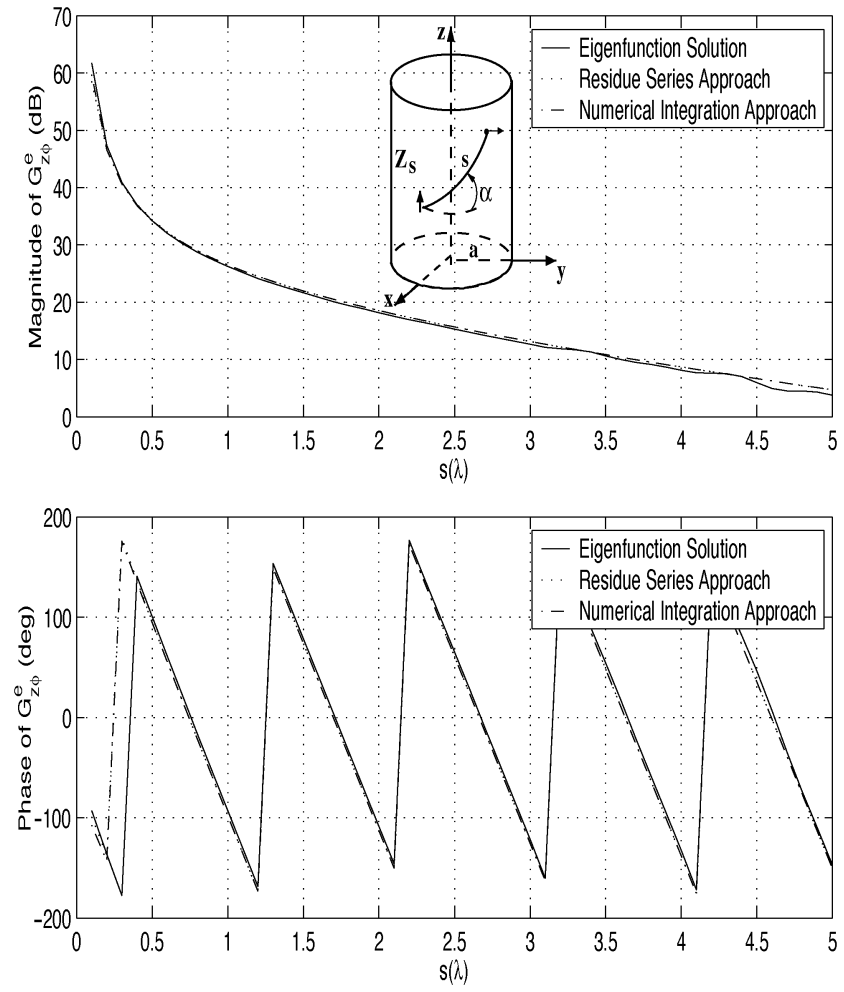

Fig. 8. Comparison of the magnitude (in $\mathrm{dB}$ ) and phase of the $G_{z \phi}^{e}$ versus separation $s$ obtained by the eigenfunction solution and the numerical approaches for $f=7 \mathrm{GHz}, a=5 \lambda, \alpha=45^{\circ}$, and $\Lambda=0.1$.
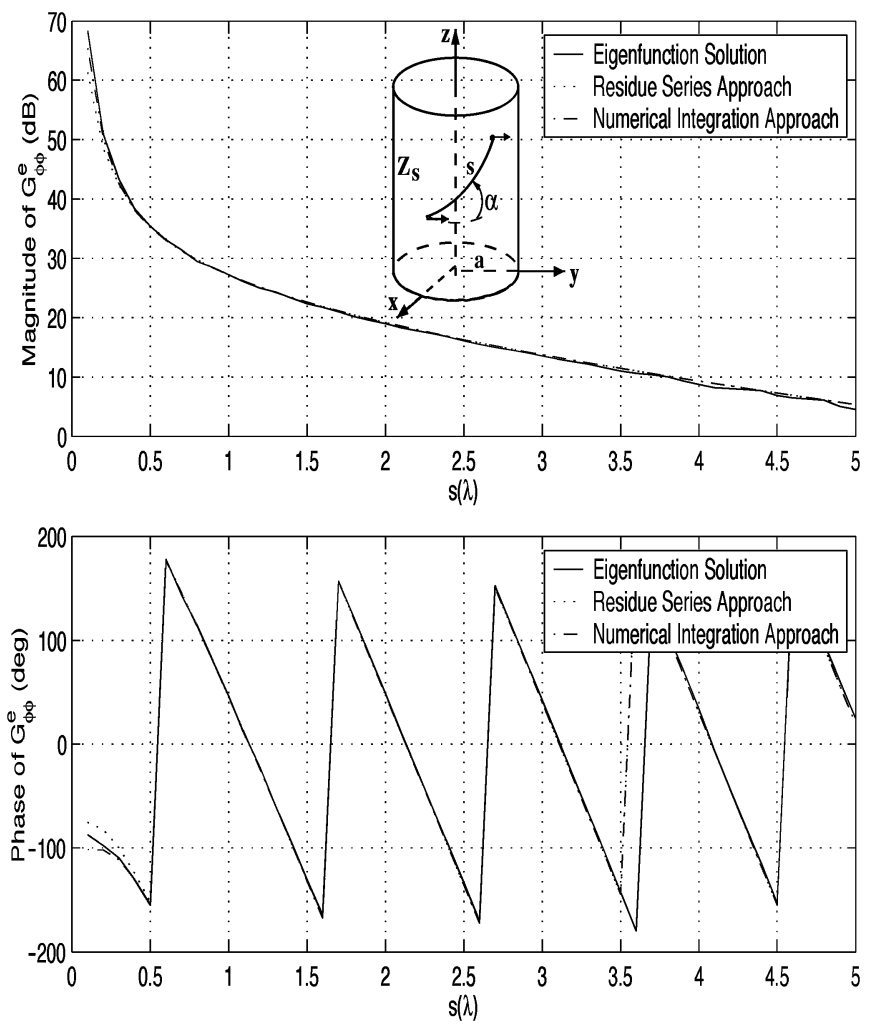

Fig. 9. Comparison of the magnitude (in $\mathrm{dB}$ ) and phase of the $G_{\phi \phi}^{e}$ versus separation $s$ obtained by the eigenfunction solution and the numerical approaches for $f=7 \mathrm{GHz}, a=5 \lambda, \alpha=45^{\circ}$, and $\Lambda=0.1$.

\section{CONCLUSION}

An alternative numerical approach is presented for the evaluation of UTD-based asymptotic solution for the nonparaxial surface fields excited by a magnetic or an electric source located on the surface of an electrically large circular cylinder with an IBC. This alternative approach is based on performing a numerical integration for the Fock-type integrals on a deformed path, which is the major burden in the evaluation of the UTD solution. The accuracy and efficiency of this approach is compared with the previously developed one presented in [1], which is based on invoking the Cauchy's residue theorem by finding the pole singularities numerically. Both approaches yield accurate results as they are compared with the eigenfunction solution in [1]. However, performing a numerical integration on the deformed path has several advantages over the residue series approach, such as having an easier formulation and less computational time. Having these advantages makes numerical integration approach more appealing than residue series approach for the evaluation of the UTD-based asymptotic solution for the surface fields excited on an electrically large circular cylinder with an IBC.

\section{ACKNOWLEDGMENT}

The authors wish to thank the reviewers for their helpful comments on this paper.

\section{REFERENCES}

[1] C. Tokgöz, "Asymptotic high frequency analysis of the surface fields of a source excited circular cylinder with an impedance boundary condition," Ph.D. dissertation, Dept. of Electrical Engineering, Ohio State Univ., Columbus, 2002.

[2] P. Munk, "A uniform geometrical theory of diffraction for the radiation and mutual coupling associated eith antennas on a material coated convex conducting surface," Ph.D. dissertation, Dept. of Electrical Engineering, Ohio State Univ., Columbus, 1996.

[3] P. E. Hussar and E. M. Smith-Rowland, "An asymptotic solution for boundary-layer fields near a convex impedance surface," J. Electromagn. Waves Appl., vol. 16, no. 2, pp. 185-208, 2002.

[4] G. Hasserjian and A. Ishimaru, "Excitation of a conducting cylindrical surface of large radius of curvature," IRE Trans. Antennas Propag., vol. AP-10, pp. 264-273, May 1962.

[5] Z. W. Chang, L. B. Felsen, and A. Hessel, Surface ray methods for mutual coupling in conformal arrays on cylinder and conical surfaces Polytechnic Inst. of New York, 1976, Final Rep., Contract N00123-76-C-0236.

[6] S. W. Lee and S. Safavi-Naini, "Approximate asymptotic solution of surface field due to a magnetic dipole on a cylinder," IEEE Trans. Antennas Propag., vol. AP-26, pp. 593-598, Jul. 1978.

[7] J. Boersma and S. W. Lee, Surface field due to a magnetic dipole on a cylinder: Asymptotic expansion of exact-solution Electromagnetics Lab., Dept. of Electrical Engineering, Univ. of Illinois, Rep. EM78-17, Dec. 1978, prepared under Contract N00019-78-C-0064 for Naval Air Systems Command.

[8] P. H. Pathak and N. Wang, "Ray analysis of mutual coupling between antennas on a convex surface," IEEE Trans. Antennas Propag., vol. AP-29, pp. 911-922, Nov. 1981.

[9] T. S. Bird, "Comparison of asymptotic solutions for the surface field excited by a magnetic dipole on a cylinder," IEEE Trans. Antennas Propag., vol. AP-32, pp. 1237-1244, Nov. 1984.

[10] — - "Accurate asymptotic solution for the surface field due to apertures on a cylinder," IEEE Trans. Antennas Propag., vol. AP-33, pp. 1108-1117, Nov. 1984.

[11] R. G. Rojas and Z. Al-hekail, "Generalized impedance/resistive boundary conditions for electromagnetic scattering problems," Radio Sci., vol. 24, pp. 1-12, Jan.-Feb. 1989. 
[12] R. Paknys and N. Wang, "High -frequency surface field excited by a magnetic line source on an impedance cylinder," IEEE Trans. Antennas Propag., vol. 35, pp. 293-298, Mar. 1987.

[13] R. J. Pogorzelski, "On the high-frequency asymptotic evaluation of the potentials of elemental sources on an anisotropic impedance cylinder," Radio Sci., vol. 31, pp. 389-399, Mar.-Apr. 1996.

[14] C. Tokgöz and R. J. Marhefka, "Surface field excitation by a magnetic point source on an impedance cylinder," in IEEE Antennas Propag. Symp. Dig., Jul. 1999, vol. 4, pp. 2834-2837.

[15] C. Tokgöz, P. H. Pathak, and R. J. Marhefka, "An asymptotic solution for the surface magnetic field within the paraxial region of a circular cylinder with an impedance boundary condition," IEEE Trans. Antennas Propag., vol. 53, pp. 1435-1443, Apr. 2005.

[16] V. B. Ertürk and R. G. Rojas, "Efficient computation of surface fields excited on a dielectric coated circular cylinder," IEEE Trans. Antennas Propag., vol. 48, pp. 1507-1516, Oct. 2000.

[17] P. Persson and R. G. Rojas, "High frequency approximation for mutual coupling calculations between apertures on a PEC circular cylinder covered with a dielectric layer: non-paraxial region," Radio Sci., vol. 38, no. 4, pp. 18-1-18-4, Jul./Aug. 2003.

[18] P. Persson, B. Thors, and R. G. Rojas, An improved numerical approach for surface field calculations on large dielectric coated circular cylinders Division of Electromagnetic Theory, Royal Inst. of Technology, TRITA-TET 03-4, Jun. 2003.

[19] — - "An improved algorithm for surface field calculations on large dielectric covered circular cylinders using asymptotic techniques," in 2003 IEEE Int. Antennas Propag. Symp. USNC/CNC/URSI Meeting, Columbus, OH, Jun. 22-27, 2003.

[20] R. F. Harrington, Time-Harmonic Electromagnetic Fields. New York: McGraw-Hill, 1961.

[21] L. W. Pearson, "A scheme for automatic computation of Fock-type integrals," IEEE Trans. Antennas Propag., vol. AP-35, pp. 1111-1118, Oct. 1987.

[22] N. A. Logan and K. S. Yee, "A simple expression for propagation constants associated with a reactive convex surface," IRE Trans. Antennas Propag., vol. AP-10, p. 103, Jan. 1962.

[23] D. A. Hill and J. R. Wait, "Ground wave attenuation function for a spherical earth with arbitrary surface impedance," Radio Sci., vol. 15, pp. 637-643, May-Jun. 1980.

[24] L. B. Felsen and K. Naishadham, "Ray formulation of waves guided by circular cylindrical stratified dielectrics," Radio Sci., vol. 26, pp. 203-209, Jan.-Feb. 1991.

[25] J. B. Keller, S. I. Rubinow, and M. Goldstein, "Zeros of Hankel functions and poles of scattering amplitudes," J. Math. Phys., vol. 4, no. 6, pp. 829-832, Jun. 1963 .

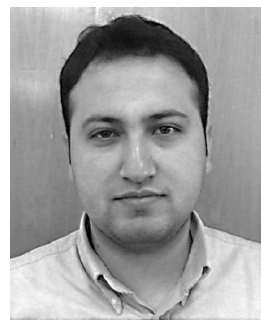

Burak Alisan received the B.S. degree from Bilkent University, Ankara, Turkey, in 2003, where he is currently pursuing the M.S. degree.

$\mathrm{He}$ has been with Aselsan Electronics Inc., Ankara, Turkey, as a radio-frequency and microwave design engineer since 2003. His research interests include application of numerical methods and asymptotic high-frequency techniques to radiation and mutual coupling problems associated with cylindrical structures.

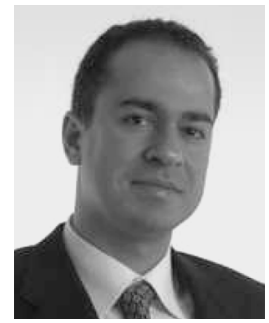

Vakur B. Ertürk (M'00) received the B.S. degree in electrical engineering from the Middle East Technical University, Ankara, Turkey, in 1993 and the M.S. and Ph.D. degrees from The Ohio State University, Columbus, in 1996 and 2000, respectively.

$\mathrm{He}$ is currently an Assistant Professor with the Electrical and Electronics Engineering Department, Bilkent University, Ankara. His research interests include the analysis and design of planar and conformal arrays, active integrated antennas, and scattering from and propagation over large terrain profiles, as well as metamaterials.

Dr. Ertürk has served as the Secretary/Treasurer of IEEE Turkey Section as well as the Turkey Chapter of the IEEE Antennas and Propagation, Microwave Theory and Techniques, Electron Devices, and Electromagnetic Compatibility Societies.

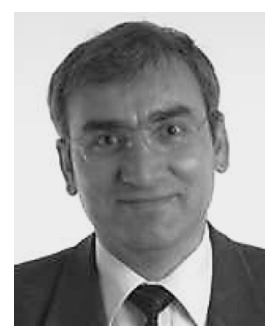

Ayhan Altintas (S'82-M'87-SM'93) received the B.S. and M.S. degrees from the Middle East Technical University (METU), Ankara, Turkey, in 1979 and 1981, respectively, and the Ph.D. degree from The Ohio State University, Columbus, in 1986.

From 1981 to 1987 , he was with the ElectroScience Laboratory, The Ohio State University. Currently, he is a Professor and Chair of Electrical Engineering at Bilkent University, Ankara. He has been a Research Fellow and Guest Professor at Australian National University, Canberra, Australia; Tokyo Institute of Technology, Japan; Technical University of Munich, Germany; and Concordia University, Montreal, PQ, Canada. His research interests include high-frequency and numerical techniques in electromagnetic scattering and diffraction, propagation modelling and simulation, and fiber and integrated optics. He has served on many university committees and was the Associate Provost of Bilkent University for 1995-1998. He is the National Chair of URSI Commission B.

Dr. Altintas is a member of Sigma Xi and Phi Kappa Phi. He was Chairman of IEEE Turkey Section for 1991-1993 and 1995-1997. He is the Founder and first Chair of the IEEE AP/MTT Chapter in the Turkey Section. Dr. Altintas is a Fulbright Scholar and an Alexander von Humboldt Fellow. He received the ElectroScience Laboratory Outstanding Dissertation Award in 1986, the IEEE Outstanding Student Branch Counselor Award in 1991, a Research Award from the Prof. Mustafa N. Parlar foundation of METU in 1991, and a Young Scientist Award from the Scientific and Technical Research Council of Turkey (Tubitak) in 1996. He received the IEEE Third Millennium Medal. 